\title{
Der Elektropflug als „Medienereignis” im Deutschen Kaiserreich
}

\author{
Zur Geschichte einer wirtschaftlich gescheiterten aber \\ propagandistisch erfolgreichen Innovation
}

\author{
Von Reinhold Bauer
}

\section{Überblick}

Im späten 19. und frühen 20. Jahrhundert brachte es der damals neue Elektropflug in Deutschland zu einiger Prominenz. Die besondere Aufmerksamkeit für diese neue Bodenbearbeitungstechnologie hatte dabei weniger mit ihren technischen Eigenschaften als vielmehr mit ihrer Wahrnehmung bzw. Deutung zu tun. Der Pflug wurde von seinen Befürwortern entweder als eine die traditionelle ländliche Gesellschaft revolutionierende oder - insgesamt verbreiteter - als stabilisierende Technologie wahrgenommen. Es handelte sich beim Elektropflug um ein ,soziotechnisches Artefakt", mit dem explizit konkrete gesellschaftliche Zielvorstellungen verknüpft wurden und das insbesondere im preußischen Osten gesellschaftsprägend bzw. -stabilisierend wirken sollte. Die öffentliche Diskussion um die gesellschaftliche Wirkung des Elektropfluges verselbständigte sich dabei im späten Kaiserreich soweit, dass die tatsächlichen Voraussetzungen für seinen Einsatz, vor allem die unzureichende Elektrifizierung gerade im ländlichen Ostelbien, zeitweilig aus dem Blick gerieten.

\begin{abstract}
In the late 19th and the early 20th century the then new electric ploughs attained a remarkable level of public awareness in the German Empire. This was not primarily due to their actual technical properties but to their perception and interpretation under the given economic and social conditions. The proponents of electric ploughing saw them as a technology with the potential to revolutionize or - more common - to stabilize traditional rural society. In this sense, electric ploughs were „sociotechnical artefacts“ explicitly linked with concrete social objectives. In particular, they were meant to have a consolidating effect on changing rural society in the eastern provinces of the Prussian monarchy. In the years prior to World War I the public discussion on the social effects of electric ploughs intensified to such an extend that the actual prerequisites for their use, especially the insufficient electrification in rural Prussia, temporarily dropped out of focus.
\end{abstract}


Im Jahre 1900 schrieb der Rittmeister a.D. und Rittergutsbesitzer Paul Mack zu Althof-Ragnit: „Daß die Elektrotechnik dazu berufen ist, in erster Linie unsern Ackerbau wieder rentabel zu machen, darüber kann ein Zweifel nach dem gewaltigen Aufschwung dieses neuen Gebietes menschlicher Forschung nicht mehr bestehen. " ${ }^{\text {II }}$ Folgenden soll es um eine im späten 19. Jahrhundert entwickelte neue Bodenbearbeitungstechnologie gehen, nämlich um den Elektropflug. ${ }^{2}$ Elektropflüge können in wirtschaftlicher Hinsicht als gescheitert gelten, waren aber, was ihre öffentliche Wahrnehmung und Wirkung im späten Deutschen Kaiserreich anbelangte, sehr erfolgreiche Maschinen. Die vermeintlich revolutionären neuen Kraftpflüge wurden seit den 1880er Jahren von verschiedenen deutschen Herstellern angeboten und brachten es vor allem im ersten Jahrzehnt des 20. Jahrhunderts zu einiger Prominenz.

Zunächst zur Technik selbst: Elektropflüge wurden in unterschiedlichen Ausführungen gebaut, am üblichsten war jedoch eine Bauart, die als Einmaschinensystem bezeichnet wurde. Dieses bestand aus drei Systemkomponenten, die wiederum über ein umlaufendes Drahtseil miteinander verbunden waren: erstens der so genannte Motorwagen, d.h. das Trägerfahrzeug für den das Drahtseil antreibenden Elektromotor, zweitens das am Drahtseil befestigte Ackergerät, also der Pflug selbst, und drittens der so genannte Gegenwagen, d.h. das Trägerfahrzeug für die Umlenkrolle, die notwendig ist, um das Drahtseil zum Motorwagen zurückzuführen. Während des Pflügens stand der Motorwagen an der einen, der Gegenwagen an der anderen Längsseite des Ackers. Ein Wendepflug wurde mit Hilfe des Drahtseils zwischen diesen Wagen hin und her gezogen, die Wagen selbst nach dem Pflügen einer Furche jeweils vorgerückt. Durch die Kombination von Querbewegung des Pfluges über den Acker und Längsbewegung der Wagen an dessen Seiten konnte ein Feld komplett bearbeitet werden. Der Motorwagen musste dabei über ein entsprechend langes Kabel mit der nächstgelegenen Stromleitung verbunden werden. ${ }^{3}$

1 Paul Mack zu Althof-Ragnit, Der Aufschwung unseres Landwirtschaftsbetriebes. Eine Untersuchung über den Dienst, den Maschinentechnik und Elektrizität der Landwirtschaft bieten, Königsberg i.Pr. 1900, S. 35.

2 Ein für die Beschäftigung mit Elektropflügen zentraler Aufsatz, auf den sich auch die folgenden Ausführungen immer wieder beziehen werden, ist: Edmund N. Todd, Electric Ploughs in Wilhelmine Germany: Failure of an Agricultural System, in: Hans-Joachim Braun (Hg.), Symposium on „Failed Innovations“, Social Studies of Science 22, 1992, S. 263-281.

3 Siehe u.a. Ohne Verfasser (o.V.), Hannoverscher Elektrotechniker-Verein (Vortrag des Ingenieurs Wurtzler über den elektrischen Betrieb in der Landwirtschaft), in: Elektrotechnische Zeitschrift (ETZ) 19, 1898, S. 92f.; Kurt Krohne, Die erweiterte Anwendung des elektrischen Betriebes in der Landwirtschaft, in: ETZ 29, 1908, Teil I, S. 928-935, hier S. 934f.; M. Schiller, Untersuchung elektrischer Pfluganlagen. Bericht im Auftrage der Deutschen Landwirtschafts-Gesellschaft, Geräte-Abteilung, Berlin 1903, S. 60ff.; Gustav Fischer, Die Elektrizität in der Landwirtschaft, in: ders. (Hg.), Die Entwicklung des landwirtschaftlichen Maschinenwesens in Deutschland (Festschrift zum 25jährigen Bestehen der Deutschen Landwirtschafts-Gesellschaft), Berlin 1910, S. 375-397, hier S. 389. Siehe auch Todd (wie Anm. 2), S. 267. 


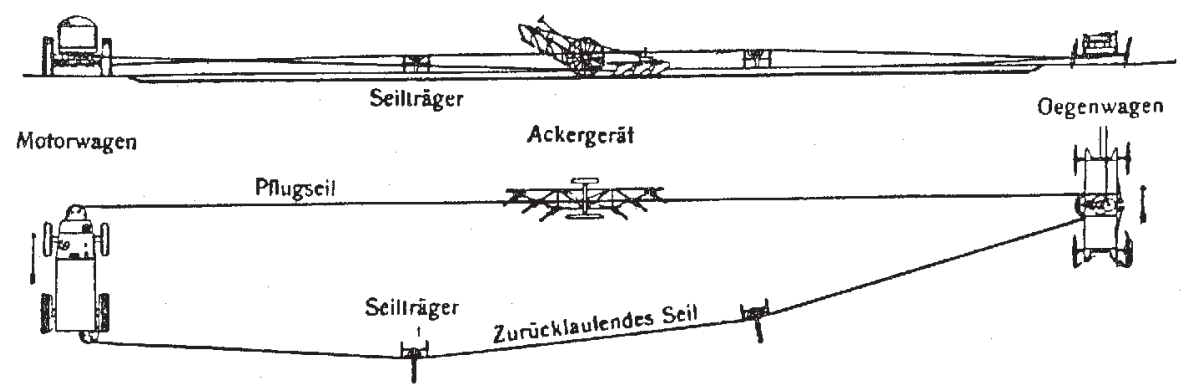

Abb. 1: Prinzip des Elektropflugeinsatzes nach dem Einmaschinensystem. Quelle: Gustav Fischer, Die Elektrizität in der Landwirtschaft, in: ders. (Hg.), Die Entwicklung des landwirtschaftlichen Maschinenwesens in Deutschland, Berlin 1910, S. 375-397, hier S. 389.

In Deutschland beschäftigte sich seit den frühen 1880er Jahren eine Reihe von Unternehmen mit der Entwicklung und Erprobung von Elektropflügen. Die beteiligten Firmen lassen sich dabei zwei großen Gruppen zuordnen, nämlich zum einen der elektrotechnischen Industrie und zum anderen dem Maschinen- bzw. Landmaschinenbau. Das eigentliche „Pionierunternehmen“ war ohne Frage die Firma Siemens \& Halske (S\&H), da hier schon 1880 der erste deutsche Elektropflug entstand und das Unternehmen auch bis in die frühen 1910er Jahre hinein kontinuierlich an der Weiterentwicklung der neuen Technologie arbeitete.

Die AEG begann nur wenig später als S\&H mit der Entwicklung von Elektropflügen und auch die Schuckert-Werke, ab 1893 Nürnberger Elektricitäts A.G., traten bis zu ihrer Fusion mit S\&H im Jahre 1903 als eigenständiger Akteur bei der Elektropflugentwicklung auf. Unter den Firmen des Ma-

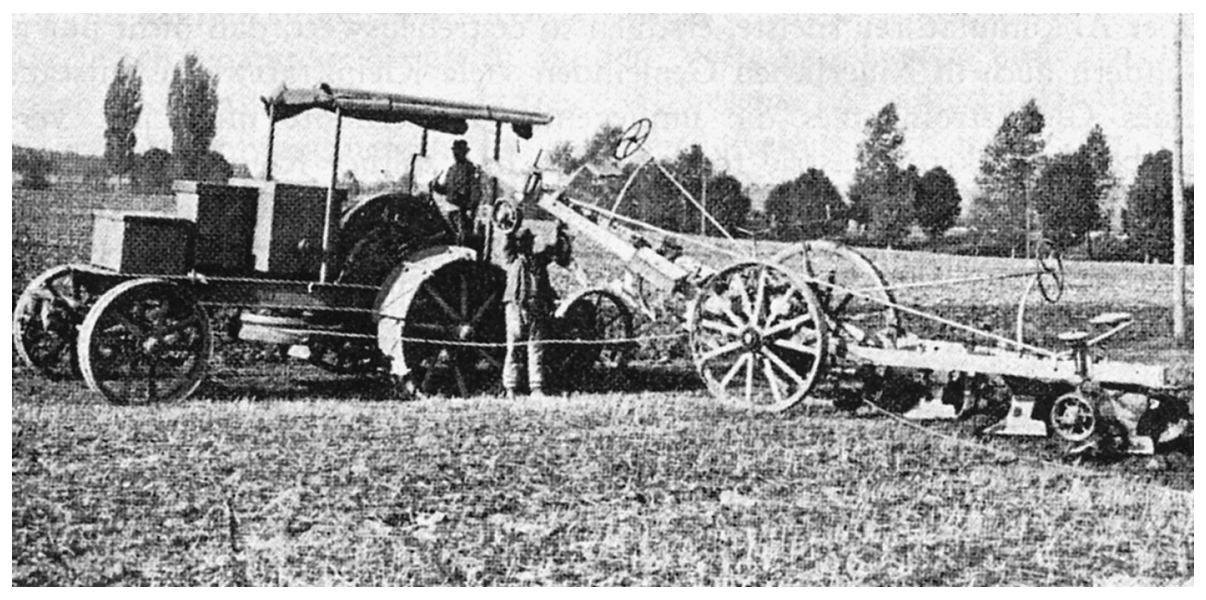

Abb. 2: Elektropflug-Motorwagen der Firma Siemens \& Halske. Quelle: M. Schiller, Untersuchung elektrischer Pfluganlagen. Bericht im Auftrage der Deutschen Landwirtschafts-Gesellschaft, Geräte-Abteilung, Berlin 1903, S. 63. 
schinen- und Landmaschinenbaus war die Hallenser Landmaschinenfabrik Zimmermann \& Co. diejenige, die sich am frühesten, nämlich bereits 1883 , dem Bau von Elektropfluganlagen zuwandte. Erst ab Mitte der 1890er Jahre kamen weitere Anbieter hinzu, darunter als mit Abstand bekanntestes und größtes Unternehmen A. Borsig in Berlin, die Landmaschinenfabriken Franz Schulte, Magdeburg, sowie die Pflugfabrik H.F. Eckert und das Maschinenbauunternehmen Emil Meyer \& Co., beide Berlin. $\mathrm{Zu}$ den Herstellern von Elektropflügen sei noch zweierlei angemerkt: Da Elektropflüge sich wie oben beschrieben aus mehreren Systemkomponenten zusammensetzten, ist mitunter kaum zu entscheiden, wer denn nun als eigentlicher Hersteller des jeweiligen Gesamtsystems zu gelten habe. So arbeiteten die Unternehmen der elektrotechnischen Industrie naheliegenderweise mit Landmaschinenfabriken zusammen und umgekehrt die Unternehmen des Maschinenbaus mit Zulieferern aus der Elektrotechnik. Allein Borsig verbaute Komponenten der Elektricitäts-A.G. Helios, von Schuckert und von S\&H. Bei allen Unternehmen spielte die Herstellung von Elektropflügen zudem rein quantitativ eine absolut untergeordnete Rolle. Es ging weniger darum, mit Elektropflügen unmittelbar Geld zu verdienen, als vielmehr darum, auf einem möglicherweise in Zukunft relevanten neuen Markt präsent zu sein. ${ }^{4}$

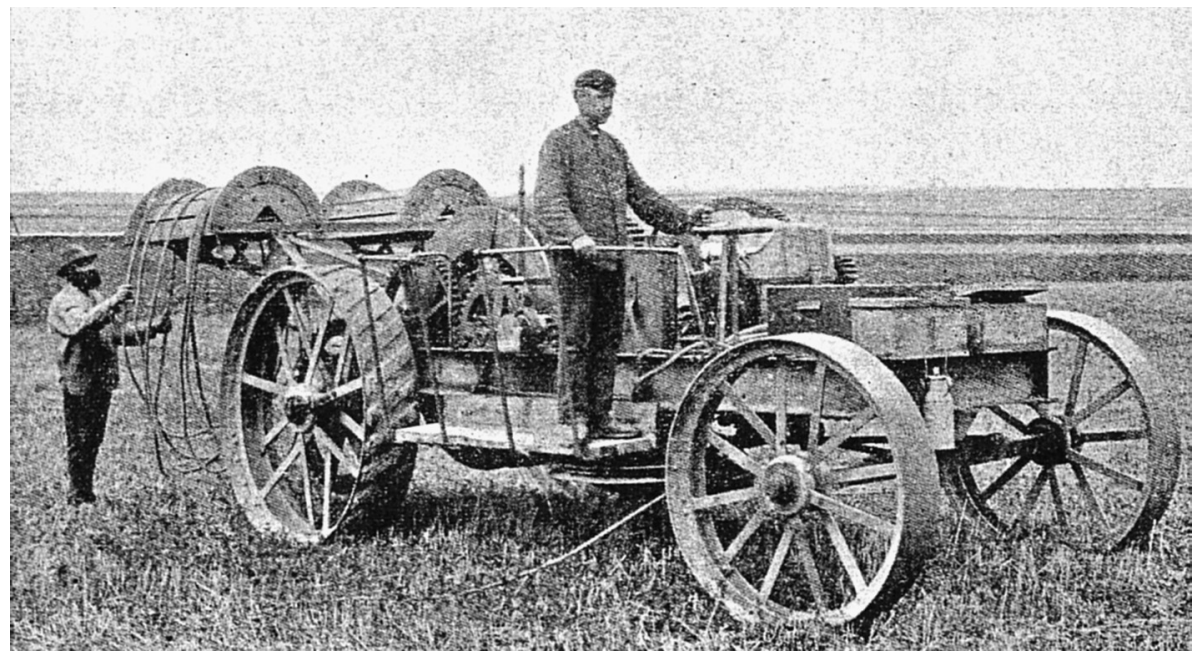

Abb. 3: Elektropflug-Motorwagen eines nicht genannten Herstellers. Quelle: A. Vietze, Die Elektrizität in der Landwirtschaft, Halle/S. 1911, S. 37.

4 Siehe u.a. Grundke, Die landwirtschaftlichen Maschinen und Geräte auf der achten Wanderausstellung der Deutschen Landwirtschafts-Gesellschaft (DLG) am 6. bis 12. Juni 1894 in Berlin, Teil III, in: Zeitschrift des Vereines Deutscher Ingenieure (ZVDI) 38, 1894, S. 1476-1482, hier S. 1481; o.V., Anwendung von Elektricität in der Landwirthschaft, in: ETZ 17, 1896, S. 39; o.V., Rundschau (Preisausschreiben der Deutschen LandwirthschaftsGesellschaft betreffend Kraftpflüge), in: ETZ 17, 1896, S. 717f.; Müllendorff, Der elek- 
Von ihrer Konstruktion wie von ihrer Verwendung her knüpften Elektropflüge jedenfalls an die schon seit Mitte des 19. Jahrhunderts gebräuchlichen Dampfpflüge an, von denen um die Wende vom 19. zum 20. Jahrhundert immerhin knapp 2.000 Stück in Deutschland in Betrieb waren. ${ }^{5}$ Allerdings boten Elektropflüge im Vergleich zu dieser älteren Konkurrenz einige wesentliche Vorteile. Zunächst waren Elektromotoren deutlich leichter als die bei Dampfpflügen eingesetzten Dampfmaschinen, was Elektropflüge insgesamt mobiler und weit universeller einsetzbar machte. Für den Elektropflug sprach zudem, dass seine Bedienung weniger personalintensiv war als die eines Dampfpfluges. Auch die bei Dampfpflügen mitunter recht aufwändige Versorgung mit den Betriebsmitteln Kohle und gegebenfalls auch Wasser entfiel. Schließlich galten Elektropflüge als potentiell weniger wartungsintensiv als die ältere Konkurrenz. ${ }^{6}$

Der Anschaffungspreis eines Elektropflugsystems lag allerdings mit etwa 30.000,- bis 40.000,- Mark nicht nennenswert unter dem eines Dampfpfluges. $\mathrm{Zu}$ den Betriebskosten des neuen Systems finden sich außerordentlich unterschiedliche Angaben, wobei das Ergebnis entscheidend von der Art der Stromerzeugung bzw. Stromversorgung abhing. Konnte für den Elektropflug z.B. Kraftstrom von einer so genannten Überlandzentrale bezogen werden, lagen seine Betriebskosten in der Regel erheblich unter denen eines Dampfpfluges. In diesem Fall war der Elektropflug, was seine Betriebskosten anbelangte, im Übrigen nicht nur dem Dampfpflug, sondern auch dem traditio-

trische Motorpflug, in: ETZ 19, 1898, S. 338-347, hier S. 338; Grundke, Die landwirtschaftlichen Maschinen und Geräte auf der 10. und 11. Wanderausstellung der DLG am 11. bis 15. Juni 1896 in Stuttgart und am 17. bis 21. Juni 1897 in Hamburg, Teil I, in: ZVDI 42, 1898, S. 119-125, hier S. 119ff.; o.V., Rundschau (zu verschiedenen Elektropflug-Systemen), in: ZVDI 42, 1898, S. 1285ff., hier S. 1285; Harald Wallem, Die Elektrizität in der Landwirtschaft und deren Beziehung zu Überlandzentralen, Teil III, in: ETZ 31, 1910, S. 728-731, hier S. 728; Gustav Fischer, Entwicklung und Stand der Technik landwirtschaftlicher Maschinen, Teil III, in: ZVDI 57, 1913, S. 1263-1268, hier S. 1266; Schiller (wie Anm. 3), S. 8, 33 u. 49, Krohne (wie Anm. 3), Teil I, S. 934.

5 Zu Dampfpflügen siehe u.a. Rudolf Franke, Motorisierung der Feldarbeit. Schlepper, in: Günther Franz (Hg.), Die Geschichte der Landtechnik im 20. Jahrhundert, Frankfurt a.M. 1969, S. 16-64, hier S. 17ff.; Walter Feuerlein, Bodenbearbeitung, in: ebd., S. 119-154, hier S. 124f.; Klaus Herrmann, Die Veränderung landwirtschaftlicher Arbeit durch Einführung neuer Technologien im 20. Jahrhundert, in: Archiv für Sozialgeschichte 28, 1988, S. 203-237, hier S. 206 u. 218; Hans Haushofer, Die deutsche Landwirtschaft im technischen Zeitalter (=Deutsche Agrargeschichte, Bd. V.), Stuttgart 1963, S. 129f.; o.V., Benutzung landwirtschaftlicher Maschinen, in: ZVDI 42, 1898, S. 865ff. Hier findet sich eine umfassende Zusammenstellung der im Deutschen Reich eingesetzten landwirtschaftlichen Maschinen und ihrer geografischen Verteilung, die auf einer Erhebung des Kaiserlichen Statistischen Amtes beruht.

6 Zu den Vorteilen des Elektropfluges siehe u.a. o.V., Anwendung der Elektricität in der Landwirthschaft, in: ETZ 16, 1895, S. 35; Hannoverscher Elektrotechniker-Verein (wie Anm. 3), S. 92f.; Müllendorff (wie Anm. 4), S. 345f.; Krohne (wie Anm. 3), Teil I, S. 928 935, hier S. 932f., Teil II, S. 950-954, hier S. 950f.; Wallem (wie Anm. 4), Teil II, S. 699703, hier S. 700. 
nellen Pflügen mit - wie es so schön heißt - ,animalischer Traktion“ überlegen. ${ }^{7}$ Der Elektropflug schien daher durchaus das Potential für eine größere Verbreitung zu haben. Insgesamt handelte es sich um eine Innovation, von der sich ihre Befürworter eine Mechanisierungswelle versprachen, welche die schwindende internationale Konkurrenzfähigkeit der deutschen Landwirtschaft wieder erhöhen sollte. ${ }^{8}$

Betriebswirtschaftlich relevant wurden seine Vorteile freilich erst ab einer gewissen Betriebsgröße, wobei von einer Mindestfläche von etwa 150 ha auszugehen ist. ${ }^{9}$ Insgesamt waren es also vornehmlich landwirtschaftliche Güter, die als Abnehmer in Frage kamen. Der potentielle geografische Schwerpunkt für den Einsatz von Elektropflügen lag damit geradezu selbstverständlich in den preußischen Ostprovinzen.

Bei der Auseinandersetzung mit der Geschichte des Elektropfluges im Deutschen Kaiserreich scheint der Begriff des „Medienereignisses“ insofern gerechtfertigt, als die mediale Präsenz dieser Maschinen im späten Kaiserreich in keinem Verhältnis zu ihrer realen Existenz als technisches Artefakt stand. Die krasse Diskrepanz zwischen der Presseberichterstattung über den Elektropflug und seiner tatsächlichen Verbreitung in der deutschen Landwirtschaft ist äußerst bemerkenswert. De facto waren am Vorabend des Ersten Weltkriegs bestenfalls ein Dutzend Elektropflüge im gesamten Deutschen Reich im Einsatz, mehr waren es im Übrigen auch seit Beginn der Entwicklungsarbeiten gleichzeitig nie gewesen. ${ }^{10}$

7 Zu den Kosten, die ein Elektropflug verursachte, siehe u.a. o.V., Elektrische Kraftverteilung für landwirtschaftliche Betriebe, in: ETZ 19, 1898, S. 411; E. Fränkel, Maschinelle und elektrische Betriebe in der Landwirtschaft, in: ZVDI 48, 1899, S. 246f.; Alexander Lang, Die Rentabilität der Maschinen in der Landwirtschaft, in: ZVDI 48, 1904, S. 1043-1047, hier insbes. S. 1045; E.W. Lehmann-Richter, Elektrische Kraft- und Licht-Anlagen in der Landwirtschaft, in: ETZ 28, 1907, S. 1027-1032, hier S. 1030; Walter Reisser, Elektrische Energieversorgung ländlicher Bezirke. Bedingungen und gegenwärtiger Stand der Elektrifizierung von Landwirtschaft, Landindustrie und ländlichem Kleingewerbe, Berlin 1912, S. 19f.; A. Vietze, Die Elektrizität in der Landwirtschaft (Arbeiten der Landwirtschaftskammer für die Provinz Sachsen, H. 22), Halle/S. 1911, S. 8; Grundke (wie Anm. 4), Teil III, S. 1476-1482, hier S. 1481; Anwendung der Elektricität (wie Anm. 5), S. 35; Rundschau (wie Anm. 4), S. 1285ff.; Müllendorff (wie Anm. 4), S. 345f.; Schiller (wie Anm. 3), S. 30f. u. 48; Krohne (wie Anm. 3), Teil II, S. 950-954, hier S. 951f.; Wallem (wie Anm. 4), Teil II, S. 699-703, hier S. 702; Fischer (wie Anm. 3), S. 379f. u. 386; Todd (wie Anm. 2), S. $265 \mathrm{ff}$.

8 Zum Aspekt der internationalen Wettbewerbsfähigkeit siehe o.V., Rundschau (Die Verwendung der Elektrizität für landwirtschaftliche Zwecke), in: ZVDI 48, 1899, S. 246f.

9 Krohne (wie Anm. 3), Teil III, S. 979-984, S. 981f.; Reisser (wie Anm. 7), S. 20. Vgl. auch Hans-Joachim Rook, Maschineneinsatz und Elektrifizierung in der Landwirtschaft Brandenburgs (1870-1930), in: Heinz Reif (Hg.), Ostelbische Agrargesellschaft im Kaiserreich und in der Weimarer Republik. Agrarkrise - junkerliche Interessenpolitik Modernisierungsstrategien, Berlin 1994, S. 233-249, hier S. 236f. u. 240ff.

10 Hermann Osten, Die ländlichen Besitzverhältnisse Preußens und die Elektrizität in der Landwirtschaft, Teil II, in: ETZ 32, 1911, S. 60ff., hier S. 62; Wallem (wie Anm. 4), Teil II, S. 699-703, hier S. 699. In der bisherigen Forschung wird vermutlich gerade wegen der starken medialen Präsenz des Elektropfluges dessen tatsächliche Verbreitung bei wei- 
Dennoch wurde seit etwa Mitte der 1890er Jahre sowohl in der technischen Fachpresse wie in der landwirtschaftlichen Verbands- und der allgemeinen Publikumspresse recht regelmäßig über den neuen Pflug und seinen potentiell segensreichen Einsatz berichtet. Ihren Höhepunkt erreichte die Medienpräsenz des Elektropfluges dabei Ende des ersten Jahrzehnts des 20. Jahrhunderts.

Welche Akteure genau diese „Medienkampagne“ zugunsten des Elektropfluges inszenierten bzw. trugen, lässt sich heute nur noch zum Teil rekonstruieren. Häufig waren entsprechende Artikel namentlich nicht gekennzeichnet oder stammten von längst vergessenen Autoren. Allerdings lassen sich bestimmte Akteursgruppen identifizieren, welche die Diskussion über mehr als zwei Jahrzehnte maßgeblich prägten. Naheliegenderweise ist zunächst auf leitende (technische) Mitarbeiter der Unternehmen zu verweisen, die an der Entwicklung des Elektropfluges beteiligt waren. Durch Veröffentlichungen in der Tages- und Fachpresse wie durch Vorträge etwa in landwirtschaftlichen Gesellschaften, über die wiederum in der Presse berichtet wurde, nahm diese Gruppe Einfluss auf die Wahrnehmung des neuen Bodenbearbeitungsgerätes. Eine zweite wichtige Akteursgruppe, zu der auch der eingangs zitierte Paul Mack zu Althof-Ragnit gehörte, bildeten die Anwender oder genauer die potentiellen Anwender der neuen Technologie selbst. Es handelt sich dabei also vor allem um Gutsbesitzer, die zum Teil in der Presse, nicht selten aber auch in selbst veröffentlichten Denkschriften u.a. zum Elektropflug Stellung nahmen. Hinzu kommen als Angehörige einer dritten erkennbaren Gruppe noch einige mit Landwirtschaftsdingen befasste preußische Beamte sowie akademisch gebildete Agrarwissenschaftler, die sich öffentlich zum Elektropflug äußerten. Schließlich ist noch die vergleichsweise große, allerdings in Teilen kaum präzise von den erstgenannten Gruppen abgrenzbare vierte Akteursgruppe der festen und freien Mitarbeiter der technischen Fachpresse zu nennen. Ein Problem ergibt sich bei dieser Gruppe daraus, dass die Verfasserangaben in den Zeitschriften zum Teil so vage sind, dass eine eindeutige Zuordnung zu bzw. Abgrenzung von den anderen Gruppen schwierig ist. ${ }^{11}$

tem überschätzt. Todd etwa geht für die Zeit um 1910 von etwa 1.000 in Deutschland eingesetzten Elektropflügen aus. Reynold M. Wik schätzte 1967 sogar, dass 1918 in Deutschland 1.600 Elektropflüge in Betrieb gewesen seien. Diese Zahlen sind auf Basis der aus der zeitgenössischen Fachpresse verfügbaren Informationen keinesfalls haltbar. Siehe Todd (wie Anm. 2), S. 269; Reynold M. Wik, Mechanization of the American Farm, in: Melvin Kranzberg u. Carroll W. Pursell (Hg.), Technology in Western Civilization, Vol. II, New York, London u.a. 1967, S. 353-368, hier S. 366.

11 Es ist nicht annähernd möglich, hier einen Überblick über alle den Elektropflug betreffenden Artikel zu vermitteln. Eine gewisse Übersicht bieten allerdings die Zusammenstellungen bei Kurt Krohne, Die Elektrizität in der Landwirtschaft, in: ETZ 34, 1913, S. 350f. sowie bei Krohne (wie Anm. 3), Teil III, S. 979-984, hier S. 983f. Mit diesem recht ausführlichen mehrteiligen Bericht Krohnes aus dem Jahre 1908 beginnt im Übrigen auch 
Explizite Gegner des Elektropfluges traten im Übrigen in der Presse des späten Kaiserreichs kaum in Erscheinung. Durchaus regelmäßig meldeten sich zwar Skeptiker zu Wort, die an der Wirtschaftlichkeit des elektrischen Ackerbaus zweifelten, einen nicht eben erfolgreichen Versuch einer wirklichen „Gegenkampagne" unternahmen aber im frühen 20. Jahrhundert lediglich die Hersteller von Dampf- und Motorpflügen. ${ }^{12}$

Insgesamt beteiligte sich jedenfalls eine recht breite Gruppe von unter anderem Unternehmern, Technikern, Beamten, Akademikern und Landwirten an der „Konstruktion“ des Medienereignisses Elektropflug. Zu fragen ist dabei, wie angesichts der realwirtschaftlichen Bedeutungslosigkeit der Elektropflüge die breite mediale Aufmerksamkeit für diese neue Bodenbearbeitungstechnologie zu erklären ist.

Um einer Antwort auf diese Frage näher zu kommen, müssen zunächst einige Aspekte der Landwirtschaftsentwicklung im Kaiserreich in den Blick genommen werden. Die Landwirtschaft war im Kaiserreich bekanntermaßen

die eigentliche Hochphase der Elektropflugdiskussion. Zu der erwähnten Akteursgruppe der leitenden Mitarbeiter siehe z.B. Anwendung der Elektricität (wie Anm. 6), S. 35 (zu einer Äußerung des Prof. Budde von Siemens \& Halske); o.V., Elektrotechnischer Verein Hamburg (zu einem Vortrag des Ingenieurs Klippe zum Thema „Elektrotechnik für die Landwirthschaft"), in: ETZ 26, 1905, S. 336f.; o.V., Elektrizität in der Landwirtschaft, in: ZVDI 53, 1909, S. 477 (Bericht über einen Vortrag des Oberingenieurs Lewin im Sitzungssaal der AEG); zur Gruppe der Gutsbesitzer siehe z.B. Kuggen von Meerscheid-Hüllesem, Was unserer Provinz frommt, Königsberg 1899 (zur Mechanisierung der ostpreußischen Landwirtschaft unter dem Eindruck der „Arbeiterfrage“); M.G. Nordmann, Agrarier helft euch selbst, Berlin 1899 (Erfahrungsbericht über die Mechanisierung eines landwirtschaftlichen Gutes in Reaktion auf die herrschende „Leutenot“); Mack (wie Anm.1); zur Gruppe der Beamten und Akademiker siehe z.B. Grundke (wie Anm. 4), Teil III, S. 1476-1482; Hannoverscher Elektrotechniker-Verein (wie Anm. 3), S. 92f.; Dr. Wolff, Elektrizität in der Landwirtschaft (Zusammenfassung eines Vortrags), urspr. in der Deutschen Landwirtschaftlichen Presse 1907, hier in: ETZ 29, 1908, S. 984; Prof. Dr. Holldack, Die Verwendung der Elektrizität in der Landwirtschaft, in: ETZ 33, 1912, S. 287-290; zur Gruppe der Fachredakteure siehe z.B. Fränkel (wie Anm. 7), S. 926ff.; Wallem (wie Anm. 4), Teil II, S. 699703; Osten (wie Anm. 10), Teil II, S. 60ff. Die technische Fachpresse nahm auch immer wieder Berichte in der Publikumspresse zum Anlass, um sich selbst dem Thema zuzuwenden. Siehe z.B. o.V., Verwendung der Elektricität in der Landwirthschaft, in: ETZ 18, 1897, S. 26; o.V., Elektrischer Betrieb in der Landwirtschaft, in: ETZ 29, 1908, S. $1069 f$.

12 Zur Gruppe der erwähnten Skeptiker siehe z.B. Robert Haas, Was hat die Elektrotechnik von der Landwirthschaft zu erwarten? in: ETZ 23, 1902, S. 771-774; K. Simons, Konstruktive Entwicklung des elektrischen Pfluges, in: ETZ 32, 1911, S. 459f. u. 481. Zur Kampagne der Hersteller von Dampf- und Motorpflügen gegen die Elektrifizierung der Landwirtschaft im Allgemeinen und gegen den Elektropflug im Besonderen siehe u.a. Heinrich Lanz, Zur Frage der Überlandzentralen, in: ETZ 32, 1911, S. 891f.; o.V., Die neuesten Miss-Erfolge der Elektrizitäts-Industrie (Faksimiliertes Flugblatt), in: ETZ 32, 1911, S. 396; o.V., Das Elektrizitätsfieber auf dem Lande, in: ETZ 33, 1912, S. 1279. Auch Beate Binder stellt fest, dass sich mögliche Gegner einer Elektrifizierung des ,platten Landes“ insgesamt vor dem Ersten Weltkrieg im öffentlichen Diskurs kaum artikulierten: Beate Binder, Elektrifizierung als Vision. Zur Symbolgeschichte einer Technik im Alltag, Tübingen 1999, S. 275. 
nach wie vor ein Wachstumssektor, der es unter dem Einfluss effektiverer Bodenbearbeitungssysteme, neuer Düngemittel, verbesserter Pflanzen- und Viehzüchtung und beginnender Mechanisierung zu stark steigenden Hektarerträgen und rasch wachsender Milch- und Fleischproduktion brachte. Dennoch war nicht zu übersehen, dass das Wachstumstempo des primären Sektors deutlich hinter dem der industriellen Produktion zurückblieb. Ende der 1880er Jahre verlor die Landwirtschaft ihr gesamtwirtschaftliches Primat an die Industrie. Der Beitrag des Agrarsektors zum deutschen Nettoinlandsprodukt wurde in den folgenden Jahren von dem des gewerblichen Sektors in rasch zunehmendem Maße übertroffen, um bis zum Ersten Weltkrieg auf einen Anteil von nicht einmal mehr 25 Prozent zu schrumpfen. In einer Phase, die einerseits von einer industriellen Hochkonjunktur und andererseits von einer lang anhaltenden Strukturkrise der Landwirtschaft geprägt war, intensivierte sich bei den Landwirten das Gefühl, mit der gewerblichen Wirtschaft und den expandierenden Städten nicht mehr mithalten zu können. Der Wandel, der sich infolge beschleunigter Industrialisierung, Entfeudalisierung, aber auch zunehmender Marktintegration und Kapitalisierung der Landwirtschaft selbst in der ländlichen Gesellschaft vollzog, wurde von Bauern und insbesondere von Gutsherren als ausgesprochen krisenhaft, als destabilisierend und gefährlich empfunden. ${ }^{13}$

In Zusammenhang mit dem Elektropflug ist hier insbesondere auf die Entwicklung im großagrarisch geprägten deutschen Osten und auf die veränderte Stellung und das veränderte Verhalten der dortigen landlosen Landarbeiter zu verweisen. Diese noch zu Beginn des 19. Jahrhunderts in der Regel gutsuntertänigen Adelsbauern wurden als Folge der Agrarreformen und des vordringenden Agrarkapitalismus zunehmend zu sesshaften Tagelöhnern, deren übliches Beschäftigungsverhältnis das der freien Lohnarbeit war. Mit der Hochindustrialisierung ging allerdings eine rasch steigende Nachfrage der Industrie auch nach ungelernten Arbeitskräften einher, so dass sich die für die Landarbeiter relativ neue, zunächst eher theoretische Möglichkeit der Abwanderung in eine reale Chance verwandelte. ${ }^{14}$

13 Siehe u.a. Haushofer (wie Anm. 5), S. 192ff.; Hartmut Harnisch, Agrarstaat oder Industriestaat. Die Debatte um die Bedeutung der Landwirtschaft in Wirtschaft und Gesellschaft Deutschlands an der Wende vom 19. zum 20. Jahrhundert, in: Reif (wie Anm. 9), S. 33-50, hier S. 36; Walter Achilles, Deutsche Agrargeschichte im Zeitalter der Reformen und der Industrialisierung, Stuttgart 1993, S. 209ff. u. 286ff.; Friedrich-Wilhelm Henning, Landwirtschaft und ländliche Gesellschaft in Deutschland, Bd. 2: 1750 bis 1976, Paderborn 1978, S. 128ff. u. 171f.; Thomas Nipperdey, Deutsche Geschichte 1866-1918, Bd. 1: Arbeitswelt und Bürgergeist, München 1991, S. 195f. u 208; Hans-Ulrich Wehler, Deutsche Gesellschaftsgeschichte, Bd. 3: Von der „Deutschen Doppelrevolution“ bis zum Beginn des Ersten Weltkrieges, 1849-1914, München 1995, insbes. S. 685f. u. 832.

14 Klaus J. Bade, Einführung: Ausländer und nationale Minderheiten in Deutschland bis 1945, in: ders. (Hg.), Auswanderer - Wanderarbeiter - Gastarbeiter. Bevölkerung und Wanderung in Deutschland seit Mitte des 19. Jahrhunderts, Bd. 2, Ostfildern 1984, S. 429-432, hier S. 429; Haushofer (wie Anm. 5), S. 135ff.; Henning (wie Anm. 13), S. 151f.; Wehler (wie Anm. 13), S. 826 u. $839 \mathrm{ff}$. 
Im Kaiserreich setzte ein Zug der unterbäuerlichen Schichten gen Westen ein, der insbesondere seit den 1880er Jahren Hunderttausende ostelbischer Landarbeiter in die Industriegebiete Mittel- und Westdeutschlands führte. ${ }^{15}$ In konservativen Kreisen beobachtete man die Abwanderung vom Lande freilich mit großer Sorge und belegte das Phänomen angesichts des besonderen Wertes, den man der Landbevölkerung zuschrieb, mit dem pejorativ gefärbten Begriff der „Landflucht“ . ${ }^{16}$ Diese Klage sollte für die „Karriere“ des Elektropfluges von einiger Bedeutung sein.

Gleiches gilt für das vieldiskutierte Problem der so genannten „Leutenot“, das sich meist unmittelbar zur Klage über die „Landflucht“ gesellte und das durch den Elektropflug ebenfalls entschärft werden sollte. Mit dem Begriff der „Leutenot" bezeichnete man den durch die Abwanderung vermeintlich verursachten Arbeitskräftemangel auf dem Lande. ${ }^{17}$

Das Lamento über den Abfluss ländlicher Arbeitskraft in die städtischen Zentren gab dabei die tatsächlichen Bedingungen auf dem ländlichen Arbeitsmarkt Ostdeutschlands kaum angemessen wider. Die ostelbischen Großagrarier hatten in den 1890er Jahren längst die wirtschaftlichen Vorteile der befristeten

15 Angetrieben wurde diese Wanderungsbewegung sicher nicht zuletzt von der besseren Bezahlung ungelernter Kräfte in der Industrie. Während zu Beginn der 1890er Jahre ein Landarbeiter im Preußischen Osten etwa zwischen 1,10 und 1,20 Mark am Tag verdienen konnte, lag die Bezahlung eines Tagelöhners in der Berliner Industrie bei etwa 2,70 Mark, in den Kohlebergwerken des Ruhrgebiets konnten unter Tage sogar mehr als 3,- Mark verdient werden. Rudolf Berthold, Landwirtschaft und Saisonarbeit in den preußischen Ostprovinzen (bis 1914), in: Zeitschrift für Agrargeschichte und Agrarsoziologie 42, 1994 , S. 26-40, hier S. 27f.; Oliver Grant, Max Weber and „Die Lage der Landarbeiter im ostelbischen Deutschland“: a statistical examination, in: Jahrbuch für Wirtschaftsgeschichte, 2002, S. 63-80, hier S. 73; Werner Sesselmeier u. Bert Rürup, Langfristige Wirkung der Arbeiterimmigration auf Arbeitsmarkt, Faktorausstattung und Wachstumspfad seit der Reichsgründung, in: Jahrbuch für Wirtschaftsgeschichte, 1996, S. 11-38, hier insbes. S. $20 \mathrm{ff}$. Recht umfassend hat sich vor allem Klaus J. Bade mit dem Phänomen der Binnenwanderung von Ost nach West auseinandergesetzt, siehe Klaus J. Bade, Vom Auswanderungsland zum „Arbeitseinfuhrland“: kontinentale Zuwanderung und Ausländerbeschäftigung in Deutschland im späten 19. und frühen 20. Jahrhundert, in: ders. (Hg.), Auswanderer - Wanderarbeiter - Gastarbeiter. Bevölkerung und Wanderung in Deutschland seit Mitte des 19. Jahrhunderts, Bd. 2, Ostfildern 1984, S. 433-485, hier insbes. S. 436.

16 Zur konservativen Klage über die „Landflucht“ siehe insbes. Klaus Bergmann, Agrarromantik und Großstadtfeindschaft, Meisenheim am Glan 1970, S. $63 \mathrm{ff}$. Siehe auch Walter Achilles, Landflucht oder Landvertreibung zwischen 1850 und 1914? in: Günther Schulz (Hg.), Von der Landwirtschaft zur Industrie. Festschrift für Friedrich-Wilhelm Henning, Paderborn u.a. 1996, S. 77; Wehler (wie Anm. 13), S. 839.

17 Die Klage über die „Leutenot“ ist in zeitgenössischen Veröffentlichungen zur deutschen Landwirtschaft nahezu allgegenwärtig. Zusammenfassend dazu siehe Jens Flemming, Fremdheit und Ausbeutung. Großgrundbesitz, „Leutenot“ und Wanderarbeiter im Wilhelminischen Deutschland, in: Reif (wie Anm. 9), S. 345-360; Klaus J. Bade, ,Billig und Willig' - die ,ausländischen Wanderarbeiter' im kaiserlichen Deutschland, in: ders. (Hg.), Deutsche im Ausland - Fremde in Deutschland. Migration in Geschichte und Gegenwart, München 1992, S. 311-324, hier S. 311; ders. (wie Anm. 15), S. 436f.; Herrmann (wie Anm. 5), S. 205 u. 208. 
Beschäftigung osteuropäischer Saisonarbeiter entdeckt. Mit Hilfe dieser vor allem aus Russisch- und Österreichisch-Polen stammenden Wanderarbeiter ließen sich die arbeitsintensiven Feldarbeiten weit preiswerter erledigen als mit den ansässigen Arbeitskräften. Es war also nicht nur die Aussicht auf ein besseres Leben in der Stadt, die Teile des Landproletariats zur so genannten ,Landflucht" bewog, sondern ebenso sehr die infolge der Saisonarbeiter zunehmende Unterbeschäftigung in den Herkunftsgebieten, die zur Abwanderung zwang. ${ }^{18}$

„Leutenot" und „Landarbeiterfrage“ wurden im Übrigen nicht nur als betriebswirtschaftliche, sondern komplementär dazu auch als „soziale Frage" diskutiert. ${ }^{19}$ Die konservativen Honoratioren auf dem Lande befürchteten, die Abwanderung könnte die Stabilität von Gesellschaft und Herrschaft auf dem Lande gefährden. Zudem sah man die gen Westen Strebenden in Gefahr, unter den verderblichen Einfluss des städtischen Lebens und insbesondere der so genannten ,sozialdemokratischen Agitation“ zu geraten. Damit aber wähnte man nicht nur das ländliche Deutschland, sondern das Herrschafts- und Gesellschaftsgefüge des Kaiserreichs insgesamt in Gefahr. ${ }^{20}$

Darüber hinaus spielten die Wanderungsbewegungen noch für ein weiteres konservativ-nationalistisches Schreckensszenario eine wesentliche Rolle, nämlich für die Angst vor der so genannten „slawischen Flut“, die den preußischen Osten angeblich zu verschlingen drohte. Obwohl die Abwanderungswelle bekanntlich nicht nur die deutschen Landarbeiter erfasste, galt sie den Radikalnationalisten im Kaiserreich als eines der zentralen das „Deutschtum“ im Osten gefährdenden und daher zu bekämpfenden Probleme. Auch viele Großagrarier forderten lauthals die nationale Verteidigung des Deutschtums im Osten, was sie freilich in keiner Weise davon abhielt, sich als Agrarunternehmer bei der Beschäftigung von Landarbeitern ausschließlich an Lohnkosten zu orientieren. Diese aber sprachen eindeutig für die Beschäftigung polnischer Saisonarbeiter. Stellvertretend sei hier der Rittergutsbesitzer von Below-Saleske zitiert, der 1906 auf einer Tagung der pommerschen Landwirtschaftskammer bemerkte: „Im Sommer flotte Arbeit ... und dann fort mit der Gesellschaft! Im Winter braucht man ... nur Leute zum Viehfüttern und Dungausfahren (und) hat keine Scherereien“..21

18 Bade (wie Anm. 15), insbes. S. 437; ders. (wie Anm. 17), insbes. S. 311f.; Flemming (wie Anm. 17), passim; Grant (wie Anm. 15), S. 73; Haushofer (wie Anm. 5), S. $180 \mathrm{ff} . ;$ Henning (wie Anm. 13), S. 152f.; Herrmann (wie Anm. 5), S. 232f.; Wehler (wie Anm. 13), S. 842. Nachvollziehbar scheint in diesem Zusammenhang die Position Walter Achilles, der vor einigen Jahren fragte, ob statt des Begriffes „Landflucht“ nicht eher jener der „Landvertreibung" angemessener sei. Achilles (wie Anm. 16), S. 77-106.

19 Jörg Lichter, Die Landarbeiterfrage in einer hochindustrialisierten Region (Rheinprovinz) 1880 bis 1914, in: Zeitschrift für Agrargeschichte und Agrarsoziologie 43, 1995, S. 23-41, hier S. 23.

20 Zusammenfassend hierzu Bergmann (wie Anm. 16), S. 63ff.

21 Zit. nach Flemming (wie Anm. 17), S. 352. Ansonsten siehe Achilles (wie Anm. 13), S. 307f.; Bade (wie Anm. 15), S. 441; ders. (wie Anm. 17), S. 322; Flemming (wie Anm. 17), insbes. S. 357ff.; Haushofer (wie Anm. 5), S. 183ff.; Wehler (wie Anm. 13), S. $961 \mathrm{ff.} \mathrm{u.} 1075$. 
Bevor es nun um die Frage geht, welche Rolle die geschilderten wirtschaftlichen und gesellschaftlichen Verhältnisse auf dem Lande für den medialen Erfolg des Elektropfluges spielten, noch einige Anmerkungen zu den Interessen der Elektrizitätswirtschaft bzw. der elektrotechnischen Industrie. Wie bereits erwähnt, wurde die zentrale Elektrizitätsversorgung in Deutschland in den anderthalb Jahrzehnten vor dem Ersten Weltkrieg zunehmend auch auf ländliche Gebiete ausgedehnt. Träger dieser Expansion waren zunächst häufig Elektrizitätsgenossenschaften, wobei sich bald auch die Elektrizitätswirtschaft mit größeren Überlandzentralen ihren Anteil am ländlichen Strommarkt zu sichern versuchte. Mit der Wirtschaftlichkeit dieser frühen Versorgungsnetze war es allerdings in der Regel nicht weit her, da der insgesamt niedrige Stromverbrauch auf dem Lande einen gewinnbringenden Betrieb der Anlagen häufig nicht erlaubte. Die umfassende Elektrifizierung der Landwirtschaft schien aber ein geeigneter Ansatzpunkt, um den ländlichen Stromverbrauch insgesamt zu erhöhen und besonders, um diesen zu verstetigen. Idealerweise sollte der Verbrauch nicht nur in den Phasen des morgendlichen und abendlichen Lichtbedarfs hoch sein, sondern auch tagsüber, wenn die Glühlampen nicht brannten. ${ }^{22}$ Dem Elektropflug kam hierbei insofern eine herausgehobene Bedeutung zu, als durch seinen Einsatz ein ungleich höherer Stromverbrauch zu erwarten war als durch alle anderen in der Landwirtschaft eingesetzten Maschinen. Im frühen 20. Jahrhundert ging man bei einem etwa 500 ha großen Gut davon aus, dass der Elektropflug an bis zu 150 Tagen im Jahr eingesetzt werden und dabei etwa $40.000 \mathrm{KWh}$ Kraftstrom pro Jahr verbrauchen würde. Der zweite vergleichsweise große Verbraucher in der Landwirtschaft, die Dreschmaschine, benötigte demgegenüber nur 4.000 KWh pro Jahr, während für alle anderen elektrischen Verbraucher zusammen mit gerade mal $3.500 \mathrm{KWh}$ gerechnet wurde. ${ }^{23}$ Elektro-

22 Zur Elektrifizierung des „platten Landes“ siehe u.a. Thomas Herzig, Wirtschaftsgeschichtliche Aspekte der deutschen Elektrizitätsversorgung 1880 bis 1890, in: Wolfram Fischer (Hg.), Die Geschichte der Stromversorgung, Frankfurt a.M. 1992, S. 123-166, hier S. 127ff.; Philipp von Hugo, Der Aufbruch in ein „elektrisches Zeitalter“: Elektroindustrie und Elektrizitätswirtschaft im Deutschen Kaiserreich 1880 bis 1918, in: Manfred Ragati u. Harald Wixforth (Hg.), Wirtschaft und Energie im Wandel der Zeit. Die Geschichte der Elektrizitätsversorgung in Ostwestfalen und Schaumburg-Lippe, Köln, Weimar u.a. 1999, S. 37-48, hier S. 44ff.; Wolfgang Zängl, Deutschlands Strom. Die Politik der Elektrifizierung von 1866 bis heute, Frankfurt a.M. 1989, S. 55ff.; Binder (wie Anm. 12), S. $231 \mathrm{ff}$. Interessante Fallbeispiele bieten auch Siegfried Buchhaupt, Umlandversorgung und Energiepolitik im Raum Mainz-Wiesbaden: Das Ausgreifen des städtischen Elektrizitätswerkes Mainz in die Region, in: Helmut Böhme u. Dieter Schott (Hg.), Wege regionaler Elektrifizierung in der Rhein-Main-Neckar-Region. Geschichte und Gegenwart, Darmstadt 1993, S. 41-56; Harald Höflein, Elektrifizierung aus der Sicht der „Peripherie“: Die Stromversorgung der Umlandgemeinde Ober-Ramstadt bis 1914, in: ebd., S. 79-91.

23 Klaus Herrmann, Pflügen, Säen, Ernten. Landarbeit und Landtechnik in der Geschichte, Reinbek 1985, S. 227; Max Hupfauer, Elektrifizierung der Landwirtschaft, in: Günther Franz (Hg.), Die Geschichte der Landtechnik im 20. Jahrhundert, Frankfurt a.M. 1969, S. 
pflüge galten also als die potentiellen „Großverbraucher“ auf dem Lande und daher ist es auch verständlich, dass sie in den Kampagnen der Elektroindustrie für die Elektrifizierung der Landwirtschaft eine besondere Rolle spielten. Insbesondere die AEG und Siemens investierten erhebliche Summen sowohl in die Weiterentwicklung und Erprobung wie in die Öffentlichkeitsarbeit für die neuen Pflüge. ${ }^{24}$

Welche Folgen hatte nun all das für die Wahrnehmung des Elektropfluges? In den Presseberichten zur Elektrifizierung der Landwirtschaft wurde von den Befürwortern des Elektropfluges natürlich stets auf dessen betriebswirtschaftliche Vorteile verwiesen. Zugleich wurde er aber auch als mögliche Antwort auf die vieldiskutierten Probleme ,Landflucht“" und ,Leutenot" vorgestellt. ${ }^{25}$ Dabei argumentierten seine Promotoren entlang zweier sich im Grunde widersprechender Stränge:

Zum einen sollte die Mechanisierung des Pflügens schlicht den Arbeitskräftebedarf bei der Bestellung des Bodens senken, damit aber nicht nur die Produktionskosten, sondern auch die Abhängigkeit des Gutsherren von vermeintlich knapper Lohnarbeit verringern. Das im Vergleich zum üblichen

102-118, hier S. 104ff. Zum Problem des unzureichenden Stromverbrauchs auf dem Lande und zur unzureichenden Rentabilität ländlicher Netze finden sich ab etwa 1910 vermehrt Berichte in der ETZ. Siehe dazu insbes. auch Haas (wie Anm. 12), S. 771-774; Elektrotechnischer Verein Hamburg (wie Anm. 11), S. 336f.; o.V., Die 18te Jahresversammlung des Verbandes der Elektrotechniker (Thema: Verwendung der Elektrizität in der Landwirtschaft und ihre Beziehung zu den Überland-Kraftwerken), in: ZVDI 54, 1910, S. 1034f.; H. Brüggeln, Pauschaltarife für landwirtschaftliche Motoren, in: ETZ 33, 1912, S. 5ff., hier S. 5. Ansonsten siehe K.K. (wohl Kurt Krohne), Landwirtschaft. Erfahrungen eines Landwirtes, in: ETZ 32, 1911, S. 1278; Elektrische Kraftverteilung (wie Anm. 7), S. 411; Fischer (wie Anm. 3), S. 386 u. 397; Vietze (wie Anm. 7), S. 7f. Zu ländlichen Regionen als attraktive zukünftige Märkte für die elektrotechnische Industrie siehe o.V., Rundschau (Verwendung von Elektricität in der Landwirthschaft), in: ETZ 18, 1897, S. 229f.; M. Breslauer, Die erweiterte Anwendung des elektrischen Betriebes in der Landwirtschaft (Leserbrief), in: ETZ 29, 1908, S. 1113f.; Haas (wie Anm. 12), S. 771f.; Elektrotechnischer Verein Hamburg (wie Anm. 11), S. 336.

24 Siehe u.a. o.V. 1897 (wie Anm. 4), S. 39 u. 717f.; o.V., Elektrische Betriebe in der Landwirthschaft, in: ETZ 20, 1899, S. 183; Wallem (wie Anm. 4), Teil III, S. 728-731, hier S. 728; Osten (wie Anm. 10), S. 62. Die Elektroindustrie bemühte sich naheliegenderweise um Kontakte zu Politik und Verwaltung. Siehe etwa: Elektrizität in der Landwirtschaft (wie Anm. 11), S. 477 (hier wird über einen Vortrag im Sitzungssaal der AEG vor Mitgliedern des Reichstags und verschiedener Landtage, Vertretern von Ministerien, Landwirtschaftsgenossenschaft usw. berichtet). In diesem Zusammenhang sei auch auf entsprechende „Propagandabroschüren“ der Elektroindustrie hingewiesen, etwa: Elektrizitäts-Aktiengesellschaft (vorm. Schuckert \& Co), Die Elektricität in der Landwirthschaft, Nürnberg 1896; Allgemeine Elektricitäts-Gesellschaft, Die Elektricität im Dienste der Landwirthschaft, Berlin 1898; Siemens \& Halske, Die Elektrizität in der Landwirtschaft, Berlin 1901; Siemens-Schuckert-Werke, Die Elektrizität in der Landwirtschaft, Berlin 1904, dito Leipzig 1909.

25 Vietze (wie Anm. 7), S. 7; Fränkel (wie Anm. 6), S. 926; Reisser (wie Anm. 7), S. 2; Gustav Siegel, Die Preisbewegung elektrischer Arbeit seit 1898, München, Leipzig 1914, S. 177f. 
Pflügen mit Pferden oder Ochsen weit weniger arbeitsintensive elektrische Pflügen sollte somit dazu beitragen, den Agrarunternehmer vom Problem der „Leutenot“" zu befreien. Zum anderen wurde das elektrische Pflügen - im Übrigen völlig zu Recht - als das im Vergleich zum traditionellen Pflügen körperlich wesentlich weniger anstrengende Verfahren beworben. ${ }^{26} \mathrm{Hier}$ argumentierten seine Befürworter also scheinbar mit dem Interesse der Landarbeiter an einer Verbesserung ihrer Arbeitsbedingungen. Eingebunden war dieses Argument allerdings in die Debatte um das Phänomen der „Landflucht": Befreie man den Landarbeiter von körperlich schwerer Arbeit, dann entfiele für ihn ein wesentliches Motiv, das Land auf der Suche nach besseren Lebensbedingungen zu verlassen. ${ }^{27}$

Die inneren Widersprüche dieser „Elektropflug-Propaganda“ sind kaum zu übersehen: Das im Zusammenhang mit der „Leutenot“ betonte Rationalisierungspotential steht in offenkundigem Widerspruch zu dem im Kontext der „Landflucht“ betonten Stabilisierungspotential. Dennoch avancierte der Elektropflug vor dem Hintergrund dieser Argumentation zu einer in der Öffentlichkeit viel besprochenen Innovation ${ }^{28}$ deren zukünftige Nutzung auch von durchaus unterschiedlichen Lagern reklamiert wurde. August Bebel etwa sah im Elektropflug ein zentrales Element der vollmechanisierten kollekti-

26 Zur „Schinderei des traditionellen Pflügens“ siehe Herrmann (wie Anm. 5), S. 218.

27 Die argumentative Verbindung Elektrifizierung - Elektropflug - Leutenot - Landflucht findet sich in zahlreichen Veröffentlichungen. Siehe u.a. Müllendorff (wie Anm. 4), S. 346; Lehmann-Richter (wie Anm. 7), S. 1027; Krohne (wie Anm. 3), Teil I, S. 928-935, hier S. 928f. u. 931f.; Walther Suhge, Der Elektromotor im Kleingewerbe (auch zur Landwirtschaft), in: ETZ 30, 1909, S. 152-157, hier S. 153; Gustav Fischer, Vorwort, in: ders. (Hg.), Die Entwicklung des landwirtschaftlichen Maschinenwesens in Deutschland (Festschrift zum 25jährigen Bestehen der Deutschen Landwirtschafts-Gesellschaft), Berlin 1910, S. 1-21, hier insbes. S. 15ff.; Wallem (wie Anm. 4), Teil I, S. 671-674, hier S. 671; Reisser (wie Anm. 7), S. 2; Vietze (wie Anm. 7), S. 7; Osten (wie Anm. 10), S. 60; Siegel (wie Anm. 25), S. 177f. Vgl. auch Zängl (wie Anm. 22), S. 73; Meerscheid-Hüllesem (wie Anm. 11); L. Hübel, Die Gestaltung des landwirtschaftlichen Betriebes mit Rücksicht auf den herrschenden Arbeitermangel, o.O. 1902. Siehe auch Binder (wie Anm. 12), S. $271 \mathrm{f}$. Man mag vermuten, dass die Arbeitgeber in Reaktion auf die Verbesserung der Arbeitsbedingungen zumindest den Versuch unternommen hätten, eine niedrigere Entlohnung durchzusetzen. Offen ausgesprochen wurde ein solcher Hintergedanke aber wohlweislich nicht und der mögliche Erfolg einer solchen Strategie erscheint fraglich. Zwar wurden Mechanisierungsfortschritte in anderen Bereichen landwirtschaftlicher Arbeit (z.B. bei der Einführung von Dresch- und Mähmaschinen) wiederholt zum Anlass genommen, um mit Verweis auf die nun angenehmeren Arbeitsbedingungen Lohnsenkungen durchzusetzen, doch betraf das nur die Hilfskräfte, nicht die Maschinenbediener selbst. Beim Elektropflügen war man ohne Frage auf Bedienpersonal mit einer gewissen Mindestqualifikation angewiesen, so dass sich hier eine Lohnkostensenkung wohl „nur“ durch die Rationalisierungseffekte des mechanischen Pflügens selbst, nicht aber durch Lohnsenkungen ergeben hätte. Vgl. dazu Herrmann (wie Anm. 5), S. 234ff.

28 Vietze spricht noch 1911 von einem regelrechten „Elektrizitätsfieber“ und vom „Interesse weitester Kreise“, räumt aber hier bereits in der Rückschau „Hast und Überstürzung“ ein, die es in Zukunft zu vermeiden gelte. Vietze (wie Anm. 7), S. 7. 
ven Großlandwirtschaft der Zukunft; Theodor Herzl bezeichnete ihn 1902 in seinem utopischen Roman „Altneuland“ gar als eine der wichtigsten Erfindungen des 19. Jahrhunderts und ließ seine Helden das neue Palästina mit zunächst 300 dieser Maschinen urbar machen. ${ }^{29}$

Neben Sozialdemokraten und Zionisten waren es aber insbesondere die konservativen Multiplikatoren im preußischen Osten, die geradezu plangemäß den Elektropflug als , ihre“ Maschine entdeckten. ${ }^{30}$ Elektropflüge machten so publizistisch bzw. propagandistisch Karriere als „Hilfstruppen“ in der Auseinandersetzung um „Leutenot" und „Landflucht". Das Rationalisierungspotential des Elektropfluges galt zudem als ein Ansatzpunkt zur Reduzierung des Bedarfs an den offiziell ungern gesehenen polnischen Saisonarbeitern, womit sich nochmals der argumentative Kreis zur Germanisierungsbewegung schloss. ${ }^{31}$

$\mathrm{Ob}$ die Mechanisierung des Pflügens tatsächlich geeignet sein würde, den Saisonarbeiterbedarf zu senken, wurde von den Elektropflug-Befürwortern nicht weiter problematisiert. Das ist insofern erstaunlich, als in Wirklichkeit natürlich nicht das Pflügen - sprich das vor allem vor dem Winter erforderliche Wenden des Bodens, um diesen zu durchmischen und zu lockern sowie um die von der Ernte zurückgebliebenen Stoppelreste in die Erde zu bringen - der landwirtschaftliche Arbeitsgang war, der den größten Arbeitskräfteeinsatz erforderte. $\mathrm{Zu}$ den besonders arbeitsintensiven Tätigkeiten zählten vielmehr das Hacken des Bodens bei Kartoffel- und Rübenanbau sowie u.a. die Kartoffelernte und die Getreideernte mit der Sense. ${ }^{32}$

Der Elektropflug verdankte seine mediale Karriere nicht zuletzt seiner Funktion als unterstützendes Element im Abwehrkampf gegen den Wandel der gesamtwirtschaftlichen Struktur, gegen hohe horizontale und im Endeffekt auch vertikale Mobilität, gegen die damit einhergehende Emanzipation von Minderprivilegierten sowie gegen Urbanisierung bzw. Entagrarisierung. Schlussendlich stand er also für einen mit modernster Technologie geführten Kampf gegen die sozialökonomische und politische „Modernisierung“. In diesem Sinne führten Elektropflüge zu einer recht eigentümlichen Allianz zwischen der Elektroindustrie, also einem der industriellen Führungssektoren der Hochindustrialisierungsphase und geradezu einem Symbol für den beschleunigten Wandel, und denjenigen konservativen Kreisen, die gerade die-

29 August Bebel, Frau und Sozialismus, Stuttgart $1909^{34}$, S. $461 \mathrm{ff}$.; Theodor Herzl, „Wenn ihr wollt, ist es kein Märchen“. Altneuland/Der Judenstaat, Kronberg 1978 (1. Aufl. Leipzig 1902), S. 138f.

30 Zur entsprechenden Propaganda und ihren Trägern siehe auch Bergmann (wie Anm. 16), S. 76.

31 Todd (wie Anm. 2), S. 267f. Zum Zusammenhang Elektropflug - Saisonarbeit s. auch Krohne (wie Anm. 3), Teil I, S. 928-935, hier S. 928f.; Wallem (wie Anm. 4), Teil I, S. 671-674, hier S. 671. Vgl. auch Vietze (wie Anm. 7), S. 7; Fischer (wie Anm. 27), S. 16.

32 Achilles (wie Anm. 13), S. 278f.; Fischer (wie Anm. 27), S. 17; Herrmann (wie Anm. 5), S. $209 \mathrm{ff}$. 
sen Wandel zu verhindern suchten. Paul Mack jedenfalls, der bereits eingangs zitierte Rittmeister a.D. und Rittergutsbesitzer, entwarf ein überaus beruhigendes Bild von Landwirtschaft und ländlicher Gesellschaft nach dem Siegeszug des elektrischen Ackerbaus: ,dann wird nach den wirtschaftlichen Kämpfen des letzten Jahrzehnts ... Friede und Freude einziehen unter die sich befehdenden Berufsstände! Und ... wo bleiben dann die Gespenster von heute, die ländliche Arbeiternot und die sozialdemokratischen Umtriebe? In dem Augenblick, in welchem die Landwirtschaft prosperiert, hört die Arbeiternot von selbst auf ... und damit würde der ungesunden sozialdemokratischen Wühlerei gleichfalls der Boden entzogen." ${ }^{\text {“33 }}$

Wie stand es aber nun um die tatsächlichen kommerziellen Erfolgsaussichten des Elektropfluges? Noch am Vorabend des Ersten Weltkriegs waren nur knapp 25 Prozent des ländlichen Deutschlands elektrifiziert, wobei sich die Versorgungsgebiete im dichter besiedelten Westen und Südwesten sowie in Teilen Mitteldeutschlands, d.h. in relativer Nähe zu den industriellen Ballungsgebieten, konzentrierten..$^{34}$ Der preußische Osten hingegen - also das wichtigste potentielle Einsatzgebiet des Elektropfluges - blieb weitgehend unerschlossen, dort existierten nach wie vor vornehmlich lokale städtische Netze, die kaum ins Umland hinaus wuchsen. Eine Elektrifizierung der Gutswirtschaften war also - wenn überhaupt - nur mit Hilfe von Einzelanlagen möglich, was jedoch nur unter sehr speziellen Bedingungen auch wirtschaftlich vertretbar war. ${ }^{35}$ Anders ausgedrückt: Da, wo die Struktur der Landwirtschaft mit großen Erzeugungseinheiten und großen Ackerflächen den betriebswirtschaftlich sinnvollen Einsatz von Elektropflügen eventuell erlaubt hätte, gab es in der Regel keinen Strom, da hingegen, wo auch der ländliche Raum elektrifiziert war, verbot sich die Nutzung von Elektropflügen aufgrund der vergleichsweise kleinteiligen Betriebsstruktur.

Allerdings schien es zunächst nur eine Frage der Zeit, bis auch der für Elektropflugeinsatz geeignete Osten elektrifiziert werden würde. Der Traum vom elektrischen Ackerbau war endgültig erst ausgeträumt, als sich kurz vor dem Ersten Weltkrieg eine konkurrierende neue Technologie zu verbreiten begann: der von einer Verbrennungskraftmaschine getriebene, selbstfahrende Motorpflug. Selbst die vehementesten Befürworter des Elektropfluges gaben ab 1912 zu, dass ihre Maschine angesichts der hohen Installationskosten

33 Mack (wie Anm. 1), S. 56.

34 Todd (wie Anm. 2), S. 272. Vgl. auch Hugo Ott (Hg.), Statistik der öffentlichen Elektrizitätsversorgung Deutschlands 1890-1913, St. Katharinen 1986.

$35 \mathrm{Zu}$ den wenigen Ausnahmen, wo Elektropflüge tatsächlich auf der Grundlage von Eigenanlagen oder kleineren, häufig von mehreren Gutsbesitzern genossenschaftlich errichteten und betriebenen ländlichen Elektrizitätswerken zum Einsatz kamen, siehe Preisausschreiben der Deutschen Landwirthschafts-Gesellschaft (wie Anm. 4), S. 717f.; o.V., Elektrische Kraftübertragung für landwirthschaftliche Zwecke, in: ETZ 20, 1899, S. 737. Ansonsten siehe Schiller (wie Anm. 3), insbes. S. 30; Fischer (wie Anm. 3), S. 379; Fränkel (wie Anm. 7), S. 926. 
sowie ihrer unzureichenden Eigenbeweglichkeit mit dem leichten und mobilen Motorpflug nicht würde konkurrieren können. ${ }^{36}$ Die Nische, in der der Elektropflug tatsächlich hätte Erfolg haben können, war und blieb im späten Kaiserreich zu klein und schloss sich mit der Verbreitung des Motorpfluges endgültig.

Allerdings scheiterte die Kampagne zur Stromnutzung auf dem Lande keineswegs insgesamt: Gegen Ende der ersten Jahrzehnts des 20. Jahrhunderts fanden sich Elektromotoren etwa zum Antrieb von Milchzentrifugen, Dreschmaschinen, Pumpen, Kreissägen, Schrägaufzügen oder Zerkleinerern auf fast allen bäuerlichen Vollerwerbs-Betrieben, vorausgesetzt freilich, diese waren überhaupt ans Stromnetz angeschlossen. ${ }^{37}$

Der kommerzielle Misserfolg des Elektropfluges ist leicht zu erklären, unmittelbar nachvollziehbar und erscheint in der Rückschau geradezu vorprogrammiert. Umso erklärungsbedürftiger ist daher seine breite Befürwortung, ja sein in diesem Sinne bemerkenswerter Erfolg als „Medienereignis“. Diesen Erfolg konnten weder die objektiven Hindernisse, die seiner Verwendung im Wege standen, noch die inneren Widersprüche, welche die Argumentation seiner Befürworter durchzogen, verhindern. Was war der Grund?

Zunächst einige Überlegungen, die, mangels entsprechender Aussagen in den Quellen, nicht ganz frei von spekulativen Elementen sind: Die Aufmerksamkeit, die der Elektropflug auch über die engere landwirtschaftliche Welt hinaus erregte, hatte wohl mit dem eigentümlichen Charakter der Technologie selbst zu tun. Mit dem Elektropflug drang ja gleichsam der „Fortschritt" in seiner damals modernsten Form, der Elektrotechnik, in den Ackerbau als der nach der Jagd sicherlich archaischsten Form menschlicher Arbeit ein. Dieses Eindringen erfolgte dabei nicht etwa in einem Randbereich der Landwirtschaft, sondern in einem ihrer zentralen Arbeitsgänge, der Bodenbearbeitung mit dem Pflug. Der gebückt hinter seinem Pflug einher schreitende Mensch ist gleichsam der Archetyp des Bauern. Das Pflügen ist eben nicht irgendeine Tätigkeit, sondern kann symbolhaft für den Ackerbau insgesamt stehen..$^{38}$ Die Faszination, die vom Elektropflug ausging, ist - so

36 C. Buschkiel, Die Elektrizität in der Landwirtschaft, in: ETZ 35, 1914, S. 350; Holldack (wie Anm. 11), S. 290; Fischer (wie Anm. 4), Teil III, S. 1263-1268, hier S. 1265f.; Haushofer (wie Anm. 5), S. 195. Zum Motorpflug siehe auch Franke (wie Anm. 5), S. 20ff.; Feuerlein (wie Anm. 5), S. 125f.

37 Siehe u.a. Karl Forstreuter, Die Bedeutung der Elektrizität für die Landwirtschaft, Leipzig 1911; O. Kirstein, Elektrizität und Landwirtschaft, Berlin 1911; Walter Reisser, Steigerung der Wirtschaftlichkeit ländlicher Elektrizitäts-Versorgung, Stuttgart 1913; Vietze (wie Anm. 7), passim; Fischer (wie Anm. 3), S. 397ff.; Haushofer (wie Anm. 5), S. 195; Hupfauer (wie Anm. 23), S. 107ff.

38 Eine zeitgenössische Äußerung zur besonderen Bedeutung des Pflügens findet sich bei Krohne (wie Anm. 3), Teil I, S. 928-935, hier S. 932. Zur Wahrnehmung der Elektrizität auf dem Lande als Symbol für „Fortschrittlichkeit“ und „Modernität“ siehe Binder (wie Anm. 12), S. 268ff., insbes. S. 276. 
scheint es - nicht zuletzt auf die Gegensatzpaarung, die er verkörperte, zurückzuführen; diese machte ihn so „PR-fähig“.

Dass der Elektropflug - was seine Wahrnehmung anbelangt - zu einer vermeintlichen „Schlüsselinnovation“ hat aufsteigen können, und zwar unabhängig und sogar im Widerspruch zu seinen tatsächlichen Verwertungschancen, hatte darüber hinaus damit zu tun, dass er so hervorragend mit den wirtschaftlichen, gesellschaftlichen und letztlich auch politischen Zielen seiner einflussreichen Befürworter zu korrespondieren schien.

Bevor im Anschluss eine Art Fazit gezogen werden soll, sei schon der Vollständigkeit halber darauf hingewiesen, dass der Elektropflug keine ausschließlich deutsche Entwicklung war. Vor dem Ersten Weltkrieg fanden vor allem in den USA Versuche statt, Elektropflüge im Ackerbau einzusetzen. Die Struktur der nordamerikanischen Landwirtschaft mit ihren zum Teil riesigen Erzeugungseinheiten und Anbauflächen legte den Einsatz von Elektropflügen nahe, wobei jedoch andererseits - ganz ähnlich wie im ostelbischen Deutschland - der geringe Elektrifizierungsgrad des ländlichen Amerika die erreichbaren Erfolge stark einschränkte. Auch in den USA konnte sich der Elektropflug letztlich nicht durchsetzen und verschwand mit der Verbreitung von Motorpflügen und insbesondere Traktoren wieder von den Feldern. ${ }^{39}$

Vergleichsweise späte Elektropflugversuche sind auch für das nachrevolutionäre Russland belegt, wobei dazu bisher nur ausgesprochen dürftige Informationen vorliegen. In den frühen 1920er Jahren sind jedenfalls auf dem Versuchsgut des Moskauer Zootechnischen Instituts (Butyrsky Khutor) Elektropflüge erprobt worden. Diese Versuche müssen im Zusammenhang mit der „Neuen Wirtschaftspolitik“ (NÖP) ab 1921 und den ambitionierten Elektrifizierungsplänen für die Sowjetunion, eines der Kernelemente der geplanten beschleunigten industriellen Entwicklung, gesehen werden. De facto war die sowjetrussische Landwirtschaft zu dieser Zeit noch durch eine überwiegend kleinteilige Bodennutzungsstruktur geprägt. Die Kommunistische Partei ging allerdings davon aus, dass neue Landmaschinen - so z.B. auch der Elektropflug - einen wesentlichen Anreiz dafür schaffen könnten, die vergleichsweise ineffektive Bewirtschaftung kleiner Flächen zu überwinden. Bekanntermaßen setzte die Wirtschaftsführung aber ab 1923 auf den Einsatz von Traktoren, vor allem solchen der US-amerikanischen Firma Ford, womit auch in der UdSSR das Schicksal des Elektropfluges besiegelt war. Bemerkenswert bleibt immerhin, dass der Elektropflug auch in der frühen Sowjetunion als Fortschrittssymbol eine gewisse Rolle spielte und dort sei-

39 O.V., Verwendung des Elektromotors in landwirthschaftlichen Betrieben (zu entsprechenden Versuchen in den USA), in: ETZ 16, 1895, S. 91; K.K. (wohl Kurt Krohne), Die vorjährige Ausstellung landwirtschaftlicher Maschinen im Inlande, in: ETZ 33, 1912, S. 65ff., hier S. 65; David E. Nye, Electrifying America. Social Meanings of a New Technology, Cambridge (Mass.), New York 1991, S. 287ff., insbes. S. 295; Wik (wie Anm. 10), S. 356ff. u. 366. 
ne schon von August Bebel (s.o.) vorweggenommene Indienstnahme für eine völlig andere politische Utopie nachweisbar ist. ${ }^{40}$

Zum Medienereignis Elektropflug im Deutschen Reich bleibt zusammenfassend festzuhalten, dass für die besondere Aufmerksamkeit, die der neue Pflug im späten Kaiserreich erregte, seine technischen Eigenschaften oder sein tatsächliches Rationalisierungspotential eine eher untergeordnete Rolle spielten. Entscheidend waren vielmehr zwei wichtige Rahmenbedingungen: Zum einen die anhaltende Krise bzw. das anhaltende Krisenbewusstsein in der deutschen Landwirtschaft, zum anderen die beginnende Elektrifizierung des so genannten ,platten Landes“ in den anderthalb Jahrzehnten vor Beginn des Ersten Weltkrieges. Letztere ging mit einer wesentlich von der Elektrizitätswirtschaft initiierten Diskussion um die Nutzung von Strom in der Landwirtschaft einher, die unter anderem den Elektropflug als eine die Landwirtschaft und damit auch die traditionelle ländliche Gesellschaft revolutionierende oder - als Ansicht insgesamt weiter verbreitet - stabilisierende Technologie thematisierte. Der Elektropflug konnte infolgedessen als ein ,soziotechnisches Artefakt" wahrgenommen bzw. gedeutet werden, mit dem explizit konkrete gesellschaftliche Zielvorstellungen verknüpft wurden und das insbesondere im preuBischen Osten gesellschaftsprägend bzw. gesellschaftsstabilisierend wirken sollte.

Sein Scheitern, ja die Diskrepanz zwischen seinen kommerziellen Erfolgsaussichten und seiner Deutung, eröffnen dabei einen besonderen Blick auf die enorme Eigendynamik, die Debatten um neue Technologien entwickeln können. Das Interesse, das der Elektropflug erregte, basierte eben nicht auf seiner tatsächlichen Nützlichkeit als Ackerbaugerät, sondern auf seinem auch symbolischen Potential für die Verbreitung bestimmter gesellschaftlicher und politischer Ideen. Entscheidend dafür war eben tatsächlich sein soziotechnisches, nicht sein ökonomisches oder technologisches Potential.

Das Beispiel des Elektropfluges dokumentiert also, wie sehr neue Technologien zum „Medienereignis“ aufsteigen können, sofern sie zu den sozialen, ja kulturellen Wertvorstellungen gesellschaftlicher Meinungsführer zu

40 Leo Trotzki hatte bereits 1913 in seiner Schrift Literatur und Revolution den Elektropflug als eines der Instrumente beschrieben, dessen sich der sozialistische Mensch der Zukunft bedienen werde, um die unvollkommene ,natürliche“ Landschaft seinen Bedürfnissen entsprechend zu einer besseren „künstlichen“ umzuformen. Leo Trotzki, Literatur und Revolution, Wien 1924 (urspr. russisch 1913), S. 247ff. Ansonsten siehe u.a. R.W. Davies, The Socialist Offensive. The Collectivisation of Societ Agriculture 1929-1930 (=The Industrialisation of Soviet Russia 1), London u.a. 1980, S. 3ff. u. 382ff.; Hans-Joachim Braun, Konstruktion, Destruktion und der Ausbau technischer Systeme zwischen 1914 und 1945, in: ders. u. Walter Kaiser, Energiewirtschaft, Automatisierung, Information ( = Propyläen Technikgeschichte Bd. 5), Berlin 1992, S. 11-279, hier S. 241ff.; http:// www.stel.ru/-museum/lenin_leader_russian_socialist_society. htm, letzter Zugriff am 4.8.2006; http://www2.cddc.vt.edu/marxists/admin/intro/history/csf/other/lenin.htm, letzter Zugriff am 4.8.2006; http://trotsky.org/archive/lenin/photo/1921/043.htm, letzter Zugriff am 4.8.2006. 
passen scheinen. Folgt diesem „Hype“ der kommerzielle Erfolg, so wird es außerordentlich schwierig, bei der Suche nach den Gründen für diesen Erfolg zwischen tatsächlichen Eigenschaften oder relativer Vorteilhaftigkeit einer neuen Technologie und bloß interessengeleiteten „Rationalitätskonstruktionen“ zu unterscheiden. Das Scheitern hingegen legt den Blick auf diese „Konstruktionen“ frei und verdeutlicht damit die soziale Dimension technischer Entwicklung.

Das hier vorgestellte Fallbeispiel vermag insofern zu bestätigen, dass gerade die Auseinandersetzung mit gescheiterten Innovationen ${ }^{41}$ mitunter eine besonders umfassende und realitätsnahe Beschreibung technischen Wandels ermöglicht. Oftmals kann sie die außertechnischen Einflüsse auf den Innovationsprozess - wirtschaftliche und soziale aber auch politische und kulturelle Faktoren - deutlicher zeigen, als es die Untersuchung erfolgreicher Entwicklungen vermag. Natürlich gehört zu den für die Erforschung innovatorischer Fehlschläge konstituierenden Überzeugungen, dass Scheitern ein komplexer Prozess ist, der genauso erklärungsbedürftig ist wie Erfolg. Letztlich soll es aber darum gehen, dem Charakter technischen Wandels insgesamt auf die Spur zu kommen. Gescheiterte Innovationen bilden in diesem Zusammenhang die Anschauungsobjekte, mit Hilfe derer es gelingen kann, Technik im Entstehungsprozess zu untersuchen. Der Fehlschlag ist gleichsam das Vehikel, durch dessen Nutzung ein anderer Blick auf die Entstehungsbedingungen von Technik ermöglicht werden soll. Genau diese Möglichkeit macht es so reizvoll und gewinnbringend, sich mit Misserfolgen zu beschäftigen. Bildlich gesprochen geht es nicht nur darum, einen Blick auf die Ursachen für innovatorisches Scheitern zu werfen, mithin das Scheitern selbst zu erklären, sondern darüber hinaus darum, mit Hilfe des Scheiterns auch die Hintergründe technologischen Wandels genauer in den Blick nehmen zu können.

Anschrift des Verfassers: PD Dr. Reinhold Bauer, Helmut Schmidt Universität, Universität der Bundeswehr Hamburg, Seminar für Geschichtswissenschaft, Lehrstuhl für Neuere Sozial-, Wirtschafts- und Technikgeschichte, Holstenhofweg 85, 22043 Hamburg, E-Mail: rbauer@hsu-hh.de

41 Unter einer Innovation sei hier die erstmalige wirtschaftliche Verwertung einer neuen Problemlösung verstanden. Sie kann dann als erfolgreich bezeichnet werden, wenn es grundsätzlich gelingt, die entstandenen Entwicklungs- bzw. Innovationskosten wieder zu erwirtschaften. Bei einer gescheiterten Innovation misslingt hingegen die wirtschaftliche Verwertung. Das entscheidende Kriterium sei also der kommerzielle Erfolg oder Misserfolg einer Neuerung. Näheres zur Definition von erfolgreichen und gescheiterten Innovationen und den sich hieran anschließenden Überlegungen findet u.a. in Reinhold Bauer, Gescheiterte Innovationen. Fehlschläge und technologischer Wandel, Frankfurt a.M. 2006; ders., Der „Flop“ als Forschungsobjekt? Gescheiterte Innovationen als Gegenstand der historischen Innovationsforschung, in: Reinhold Reith, Rupert Pichler u. Christian Dirninger (Hg.), Innovationskultur in historischer und ökonomischer Perspektive. Modelle, Indikatoren und regionale Entwicklungslinien, Innsbruck, Wien u.a. 2006, S. 39-56; ders., Brauchen wir eine „Geschichte des Scheiterns"? Fehlgeschlagene Innovationen als Gegenstand der historischen Technikgeneseforschung, in: Hamburger Wirtschafts-Chronik, NF 4, 2004, S. 57-84. 